\title{
The role of viscosity in AGN outflows in relation to jet periodicities
}

\author{
G. Lanzafame ${ }^{1}$, P. Cassaro ${ }^{2}$, F. Schilliró ${ }^{2}$, V. Costa $^{3}$, G. Belvedere $^{4}$, and R. A. Zappalá ${ }^{4}$ \\ 1 INAF - Osservatorio Astrofisico di Catania, via S. Sofia 78, 95123 Catania, Italy \\ e-mail: glanzafame@oact.inaf.it \\ 2 INAF - Istituto di Radioastronomia, contrada Renna Bassa, CP 161, 96017 Noto (SR), Italy \\ 3 Dipartimento di Metologie Fisiche e Chimiche per l'Ingegneria dell’Universitá, Viale A. Doria 6, 95125 Catania, Italy \\ ${ }^{4}$ Dipartimento di Fisica e Astronomia dell’Universitá, Sezione Astrofisica, via S. Sofia 78, 95123 Catania, Italy
}

Received 28 May 2007 / Accepted 24 January 2008

\section{ABSTRACT}

\begin{abstract}
Aims. Adopting the Smoothed Particle Hydrodynamics (SPH) numerical method, we performed a grid of evolving models of a 3D axially-symmetric, viscous accretion disc around a supermassive black hole (SMBH) of $10^{6} \div 10^{9} M_{\odot}$. Such sort of simulations are typical of accretion discs in active galactic nuclei (AGN). In such disc models, we pay attention to the role of the specific angular momentum $\lambda$ and the turbulent viscosity parameter $\alpha$, according to the Shakura and Sunyaev prescription. One or two shock fronts in the radial inviscid flow develop, according to the assigned initial kinematic and thermodynamic conditions.

Methods. By fixing the initial and boundary conditions on the flow at the disc outer edge, where the total energy, the specific angular momentum $\lambda$, and the initial velocity are concerned, we find pairs of $(\alpha, \lambda)$ values determining radial periodical oscillations in the shock front. In some cases, an outflow develops from the subsonic post-shock region, close to the black hole.

Results. The link between the accretion disc and the jet refueling through the onset of a centrifugal shock front is evident. We also compare model variability periods with observed ones in the radio light curves of AGN.
\end{abstract}

Key words. accretion, accretion disks - galaxies: active - shock waves - galaxies: BL Lacertae objects: general galaxies: jets - hydrodynamics

\section{Introduction}

Collimated structures of outflowing plasma (jets) are observed in many classes of astrophysical objects: young stellar objects (Reipurth \& Bertout 1997); X-ray binaries (Mirabel \& Rodriguez 1999); planetary nebula nuclei (Lopez 1997); and AGN (Bragg et al. 1998; Ferrarese \& Ford 1999). In particular, in s.o. AGN, jets show non-continuous structures characterized by blob emission. The production of such blobs is connected with flares in the strongly variable light curves (Perlman et al. 2001). Generally, the radio light curves of AGN do not exhibit any periodicity, but the analysis of most time-extended radio light curves of some $X$ and $\gamma$ ray-loud blazars (Raiteri et al. 2001; Venturi et al. 2001) shows the signature of a longterm quasi-periodic behaviour. Some of the observed periodic variabilities may be explained by the helical-jet model (Königl \& Choudhuri 1985; Camenzind \& Krockenberg 1992; Raiteri et al. 1999; Guerra 2004; Rieger 2004), but kinematic studies of parsec-scale radio jets have provided observational evidence for helical trajectories only in a few AGN showing periodicities (Vicente et al. 1996; Tateyama et al. 1998; Gomez et al. 1999; Kellermann et al. 2004).

Direct observations show that accretion discs are a common feature for all jet-producing objects. It is widely accepted that the mechanism of mass loss through outflows and jets from the disc is directly linked to accretion processes. According to some hydromagnetic mechanisms (Blandford \& Payne 1984; Li et al. 1992; Tout \& Pringle 1996) magnetic fields provide a mechanical connection between jets and discs, which explain the production, confinement, and collimation of jets in an almost independent of that initial conditions.
In this paper, we present models where the outflows that refuel jets are intimately related to the accretion disc turbulent viscosity. The basic mechanism producing disc outflows is the accretion of gas onto a collapsed stellar object (typically a black hole (BH)). The exact physical properties are described in terms of the General Relativity field expression of the $\mathrm{BH}$ gravitational field. However the same physical effects can be also produced by a properly modified classical force (Paczinsky \& Wiita 1980) reproducing the effect of a general relativistic force within $10 \%$. An inviscid ideal gas accretes onto a $\mathrm{BH}$ coming from an extremely large distance. In such a physical process the global angular momentum is conserved and both the radial velocity and the tangential velocity increase inward. The gas heats close to the peak of the centrifugal barrier and triggers the formation of a shock. Bondi $(1944,1952)$ initially studied this kind of phenomena. There is a range of initial values of the flow angular momentum and energy, producing a steady shock, whose position is closer to the $\mathrm{BH}$ as decreases the initial angular momentum, as the Rankine-Hugoniot (RH) jump conditions are satisfied (Chakrabarti 1990; Chakrabarti \& Molteni 1993; Molteni et al. 1994). Physical viscosity, combined together with the angular momentum, produces a shock front capable of pushing out part of the accreting flow from the disc. For low viscosity values, the subsonic post-shock region, close to the $\mathrm{BH}$, develops in a radially, more-extended zone where the higher the viscosity the farther the steady shock front where the RH jump conditions are satisfied (Lanzafame et al. 1998; Chakrabarti \& Das 2004). Such a result has been pointed out in Chakrabarti \& Molteni (1995), Lanzafame et al. (1998), and in Chakrabarti \& Das (2004). Moreover, Lanzafame et al. (1998), and Chakrabarti \& Das (2004), have shown that, when the 
RH condition is not satisfied, the shock front shows a periodical behaviour instead of a stationary one.

In the following, we pay attention to the outflow periodicity by studying the behaviour of a disc model with parameters chosen to trigger a periodical oscillation of the shock front. We performed several simulations to obtain information about the combined effects of the specific angular momentum $\lambda$ and the Shakura-Sunyaev viscosity parameter $\alpha$. We verified which values of $\lambda$ at the disc outer edge (as an initial boundary condition) involve a periodic behaviour, for fixed $\alpha$ values. Hereinafter in the text, the words " $\lambda$ assigned" or $\lambda$ equal to a numerical value means $\lambda$ fixed at the disc outer edge as an initial boundary condition. Moreover, we tested the behaviour of the disc model by modifing the $\alpha$ value and keeping $\lambda$ constant. Further simulations have been performed to better determine the domain in the ( $\alpha$, ג) parameter space where periodicities and outflows occur. We found a range of parameters where oscillating shock fronts exist. Such periodical shocks lead to periodical variations of both the accretion and outflow rates. The latter variation obviously involves an oscillation of the number of particles flowing out in the jet. The synchrotron emission of a jet is related to the gas energy, to the magnetic field, and to the number of particles in the jet. Therefore, supposing that both energy and magnetic field are almost constant in time, a periodical variation of the jet particle number should show a periodical behaviour of the synchrotron emission and therefore of the radio light curve. The SPH model adopted in the simulations is non-dimensional, the scale factors of lengths and velocities being $R_{\mathrm{g}}=2 G M_{\mathrm{SMBH}} / c^{2}$ and $c$, respectively. This means that the physical quantities of the model have to be rescaled to get physical values. The paper is organized as follows: in Sect. 2, the model used in the simulations is illustrated; in Sect. 3, we report the simulations performed and the results obtained; finally, in Sect. 4, we give a qualitative explanation of the oscillations.

\section{The model}

In the SPH framework (Lucy 1977; Monaghan 1992) we carried out accretion disc models around a s.o. $\mathrm{SMBH}\left(M_{\mathrm{SMBH}}=\right.$ $\left.10^{6} \div 10^{9} M_{\odot}\right)$ in the axisymmetric case. Following Lanzafame et al. (1998), we adopted cylindrical coordinates, and assume all derivatives $\mathrm{d} / \mathrm{d} \phi$ equal to zero. In such formulation, each $\mathrm{SPH}$ particle is represented by a torus whose thickness is equal to the SPH resolution $h$. Exhaustive descriptions of cylindrical SPH, adopted to solve axially symmetric problems, can be found in Petschek \& Libersky (1993) and in Omang et al. (2006). Such a cylindrical SPH code has been applied successfully in Chakrabarti \& Molteni (1993), Molteni \& Sponholz (1994), and Molteni et al. (1994), as for accretion disc models fixing the thermodynamic and the sub-Keplerian kinematic inflow conditions at the outer boundary of the computational domain. Typically, in cartesian SPH the interpolated physical quantity $A_{i}$ is computed at the $i$ th particle position through the interpolation summation $A_{i}=\sum_{j}^{N} A_{j} W_{i j}$ calculated on $j=1, \ldots, N$ neighbour particles, where $W_{i j}$ is the interpolation Kernel. Instead in cylindrical coordinates, the same interpolation is made as $A_{i}=\sum_{j}^{N} A_{j} W_{i j} / 2 \pi X_{i}$, where $X_{i}$ is the $i$ th particle radial cylindrical coordinate. We model the non-rotating central compact object adopting the pseudo-Newtonian potential proposed by Paczynski \& Wiita (1980):

$\Phi=-\frac{1}{2(R-1)}$ where $R=\sqrt{X^{2}+Y^{2}}$ is the radial distance. $X$ and $Y$, as well as the other lengths, are in units of the Schwartzschild radius. The Lagrangian expressions for the two-dimensional fluid dynamics are: the continuity equation,

$\frac{\mathrm{d} \rho}{\mathrm{d} t}=-\rho \nabla \cdot \underline{v}$

where $\mathrm{d} / \mathrm{d} t$ is the co-moving Lagrangian derivative, $\rho$ is the density, and $\underline{v}$ the velocity; the momentum equation,

$\frac{\mathrm{d} \underline{v}}{\mathrm{~d} t}=-\frac{1}{\rho} \nabla p+\underline{g}+\frac{v_{\phi}^{2}}{X} \hat{X}-\frac{v_{\phi} v_{X}}{X} \hat{\phi}+\frac{1}{\rho} \nabla \cdot \tau$

where the pressure $p$ satisfies the perfect gas state equation $p=(\gamma-1) \rho U_{\text {th }}$, with $U_{\text {th }}$ the specific thermal energy; $g=$ $-\underline{R} / 2 R(R-1)^{2}$ is the gravitational acceleration; $\hat{r}$ is the radial direction vector; $\hat{\phi}$ is the tangential vector; and $\tau$ is the stress tensor. According to the Shakura-Sunyaev turbulent viscosity prescription (Shakura 1972; Shakura \& Sunyaev 1973), we adopted:

$\mu=\alpha \rho c_{\mathrm{s}} Z_{\mathrm{d}}$,

where $c_{\mathrm{s}}$ is the sound speed $\left(c_{\mathrm{s}}=\sqrt{\gamma p / \rho}\right)$ and $Z_{\mathrm{d}}$ is the vertical thickness, estimated from the assumption of the vertical equilibrium condition

$Z_{\mathrm{d}}^{2}=\frac{2}{\gamma} c_{\mathrm{s}}^{2} R^{2}(R-1)$

The polytropic index $\gamma$, introduced in the state equation $P=$ $(\gamma-1) \rho U_{\text {th }}$ has the meaning of a numerical parameter whose value, in principle, lies in the range between 1 and $5 / 3$, according to the kinetic theory of gases. Here, the adopted value is $\gamma=4 / 3$. This value, lower than $5 / 3$, better simulates the radiative losses in a disc model in which the radiative processes are not explicitly considered, e.g. Lanzafame \& Belvedere (2005). Usually, the lower the gas compressibility, the higher the polytropic index $\gamma$. However, the $\gamma$ value does not have any strict relation to the traditional adiabatic index given by the specific heats ratio, although $\gamma=1$ characterizes an isothermal gas. The energy equation is:

$\frac{\mathrm{d}}{\mathrm{d} t}\left(U_{\mathrm{th}}+\frac{1}{2} \underline{v}^{2}\right)=-\frac{p}{\rho} \nabla \cdot \underline{v}+\underline{v} \cdot \frac{\mathrm{d} \underline{v}}{\mathrm{~d} t}+\frac{1}{\rho} \nabla \cdot(\tau \cdot \underline{v})$

where $\mathrm{d} \underline{v} / \mathrm{d} t=-\nabla P / \rho+\underline{g}$, and $\tau \cdot \underline{v}$ is the vector resulting from the contraction of the stress tensor with the velocity vector.

The SPH method belongs to the so-called "shock-capturing" methods since the role of the artificial viscosity, in inviscid hydrodynamics, is to allow shocks to satisfy the Euler equation when discontinuities (shock or contact discontinuities) are present. In the case of "shock tracking" methods, a complete steady solution requires the equations of energy, angular momentum, and mass conservation to be supplemented by transonic conditions at some critical points and RH conditions at an unknown weak shock location (Chakrabarti 1989, 1990). Therefore such methods involve two different codes to solve Euler equations as for inviscid hydrodynamics. The first one is the adopted integration method to solve the fluid-dynamics partial differential equations without discontinuities, while the second one is a code explicitly involving the RH jump conditions for shock cases. In "shock-capturing" methods, while the introduction of a small artificial viscosity term (Monaghan 1992) does not formally modify the original hyperbolic Euler equations, it allows a practical handling of flow discontinuities. 
We set the particle injection (and the outer edge initial and boundary conditions on the flow) at $D=30$ Schwarzschild radii. Time, angular momentum, and energy per unit mass have scale factors of $2 G M_{\mathrm{SMBH}} / c^{3}, 2 G M_{\mathrm{SMBH}} / c$ and $c^{2}$, respectively. In this simulation, all quantities involving the mass are considered per unit mass. The SPH resolving power is $h=0.3$. We set the initial values of the radial velocity $v_{X}$ of injected particles as $v_{X}=0.1$ and the initial specific thermal energy as $U_{\text {th }}=1.08 \times 10^{-2}$. We chose these values to have the initial total energy of particles $\left(E=v_{X}^{2} / 2+\lambda 2 / 2 r^{2}+c_{\mathrm{s}}^{2} /(\gamma-1)+\Phi\right)$ equal to zero for $\lambda=1.6$, according to the total energy calculations reported in Molteni et al. (1994). Notice that since $\lambda=X v_{\phi}, \lambda=1.6$ in non-dimensional units means $\lambda=2.37 \times 10^{-38} M_{\text {SMBH }}$ cgs. At 1.2 Schwarzschild radii, corresponding to the fixed inner disc radius, particles are simply eliminated, simulating the free downfall onto the black hole.

The disc outer space is considered empty, freely crossed by particles ejected from the two disc surfaces. Of course, the value of 30 Schwarzschild radii for the outer boundary radial distance in principle does not coincide with the distance of the physical disc outer edge, which is realistically much further away, but it is far enough from the inner region for the purposes of this investigation.

The ratio of the linear dimension of the computational domain to the resolution length $D / h=100$. This value represents, at least in $1 \mathrm{D}$, a typical order of magnitude, being indeed the number of finite difference elements dividing any spatial integration domain to solve any system of partial differential equations in fluid dynamics. In $3 \mathrm{D}$, such a choice produces $100^{3}=10^{6}$ cells in which SPH particles move around the SMBH. A small modification of the resolution length by a factor $0.5 \div 2$ does not make any difference in resolving disc structures whose linear dimensions are of the order of magnitude or larger than the adopted $h$. Therefore, the assumed $h=0.3$ looks like a good choice to resolve the described disc and outflow structures. The adopted resolution length is also appropriate since the number of neighbour particles for each particle varies on the average between 15 and 50 in axially symmetric 3D. This means that such numbers, in full $3 \mathrm{D}$, correspond to $15^{3 / 2} \simeq 58$ and $50^{3 / 2} \simeq 353$, respectively. These evaluations show that the number of neighbour particles is high enough that any adaptive technique is not strictly required, and that the $3 / 2$ exponent is the numerical conversion factor to get numerical evaluations in full $3 \mathrm{D}$, also for the total number of particles. Moreover, we resolve turbulence quite well in any direction, since the assumed resolution length is lower than the minimum scale-length $L$ of turbulence necessary to get a Reynolds number $R \geq 100$. In fact, according to the adopted viscosities, we have $L \geq 1$ and $L \geq 20$, in supersonic and in subsonic regimes, respectively. Instead, the Reynolds number calculated on the whole integraton domain of 30 Schwarztschild radii is $R \sim 1.2 \times 10^{4}$ and $R \sim 10^{3}$ for supersonic and subsonic flows respectively, according to the Mach number evaluation of our results reported in Sect. 3 .

\section{Results}

In Lanzafame et al. (1998), simulations have been carried out with $\lambda=1.6$ and $0 \leq \alpha \leq 1.5 \times 10^{-3}$. These simulations refer to a stationary shock front development due to a centrifugal barrier formed during accretion onto a SMBH. In that paper, Lanzafame et al. observed a periodic oscillation of the outflow for $\alpha=1.5 \times$ $10^{-3}$, which correlated to the presence of an oscillation of the shock front. The outflow, freely moving toward the outer space mainly in the vertical direction, increases if higher $\alpha$ values are
Table 1. Values of the specific angular momentum and physical viscosity parameter for each performed simulation: Col. [1] ID code of the simulation; Col. [2] specific angular momentum $\lambda$; Col. [3] viscosity parameter $\alpha$; Col. [4] $\mathrm{P}=$ single pulsed outflow and single periodic radial shock; $\mathrm{N}=$ no outflow despite a single periodic radial shock and $\mathrm{Y}=$ outflow with two (or several) periodic radial shocks.

\begin{tabular}{cccc}
\hline \hline $\mathrm{ID}$ & $\lambda$ & $\alpha\left(\times 10^{-3}\right)$ & Outflow type \\
\hline $\mathrm{A}$ & 1.5 & 8.0 & $\mathrm{Y}$ \\
$\mathrm{B}$ & 1.55 & 2.0 & $\mathrm{~N}$ \\
$\mathrm{C}$ & 1.55 & 4.0 & $\mathrm{P}$ \\
$\mathrm{D}$ & 1.55 & 8.0 & $\mathrm{Y}$ \\
$\mathrm{E}$ & 1.575 & 2.0 & $\mathrm{~N}$ \\
$\mathrm{~F}$ & 1.6 & 2.0 & $\mathrm{P}$ \\
$\mathrm{G}$ & 1.6 & 2.5 & $\mathrm{Y}$ \\
$\mathrm{H}$ & 1.65 & 0.5 & $\mathrm{~N}$ \\
$\mathrm{I}$ & 1.65 & 1.0 & $\mathrm{P}$ \\
$\mathrm{J}$ & 1.65 & 1.5 & $\mathrm{Y}$ \\
$\mathrm{K}$ & 1.65 & 2.0 & $\mathrm{Y}$ \\
$\mathrm{L}$ & 1.7 & 0.0 & $\mathrm{P}$ \\
$\mathrm{M}$ & 1.7 & 2.0 & $\mathrm{Y}$ \\
$\mathrm{N}$ & 2.0 & 2.0 & $\mathrm{Y}$ \\
\hline
\end{tabular}

adopted. The shock front develops, comes out from the SMBH, smooths out, and then restores.

In order to examine the conditions triggering the oscillation, in this paper we performed several simulations adopting parameters different, for each model, from those used in Lanzafame et al. (1998). We fixed all parameters but $\lambda$ and $\alpha$, as remarked in Sect. 2. The values of adopted parameters for each simulation are reported in Table 1.

In all simulations, a single or a pair of shock fronts come out. In most of the simulations shocks show a periodical motion. The $\lambda$ and $\alpha$ values adopted in the simulations are plotted in Fig. 1. In the same figure, a boundary line separating two domains is drawn. This boundary is determined by interpolating the four $(\alpha, \lambda)$ pairs relative to $\mathrm{C}, \mathrm{G}, \mathrm{J}$, and $\mathrm{M}$ models of Table 1 . We note that for $\lambda \geq 1.7$, the oscillating shock front is present even for an inviscid flow, whilst the turbulent viscosity would be fundamental to trigger the oscillation if lower $\lambda$ values were adopted. In Fig. 1 the values of $\lambda$ and $\alpha$ in the shadowed region involve oscillating shock fronts and outgoing gas flows. Below the shadowed domain the shock is stationary and no outflows appear.

In Fig. 2, the outflow rate, the accretion rate, and the number of disc particles are plotted versus time for those simulations showing an outflow. Three models (B, E and H of Table 1), outside the shadowed region of Fig. 1, show a stationary shock front without any outflow. For this reason, they do not appear in Fig. 2.

Simulations with $(\alpha, \lambda)$ parameters on the boundary line of Fig. 1 show a regularly pulsed outflow, whose period increases, adopting lower $\lambda$ values. In these simulations (models $\mathrm{C}, \mathrm{F}, \mathrm{I}$, L of Fig. 2), a single shock front develops, showing periodical radial oscillations. An example of this behaviour is reported in Fig. 3, where twelve snapshots of model F, taken at different phases within one oscillation period, are plotted. Notice that the shock front comes out, moves outward, disrupts and restores periodically. Instead, models below the boundary line (see Fig. 2; models $\mathrm{B}, \mathrm{E}$ and $\mathrm{H}$ ) show a stationary shock front without any outflow, whilst models above the boundary line (shadowed domain) show a pair of oscillating shock fronts and a periodical outflow with no interruption.

Simulations with $\lambda$ and $\alpha$ in the shadowed region of Fig. 1 (models A, D, G, J, K, M, N) have a more complicated 


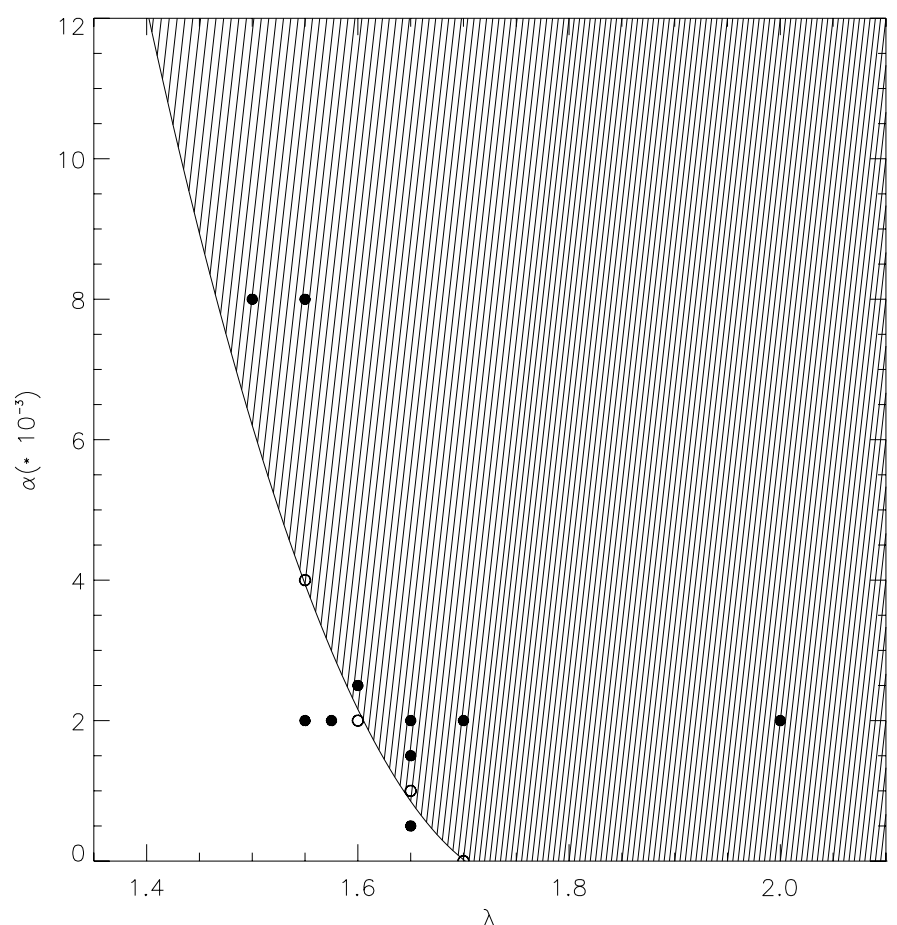

Fig. 1. $(\alpha, \lambda)$ parameter space for the simulations carried out. Each simulation is represented by a circlet, and corresponds to a pair of values of the viscosity parameter and angular momentum $(\alpha, \lambda)$. Empty circlets refer to the four boundary disc models showing single shocks and pulsed outflows. The boundary line is the limit for $(\alpha, \lambda)$ pairs to produce an oscillating shock and an outflow. Inside the shaded domain both outflows and shocks are periodical. Outflows are supported by higher $\alpha$ viscosity parameters when adopting lower initial $\lambda$ at the disc outer edge as a boundary condition.

behaviour. These models show two shock fronts that form, radially move outward, degrade, and restore in a way that is difficult to synthesize in few images, as was done in Fig. 3 for model F.

The outflow rates of our simulations show a behaviour very similar to the radio light curves of blazars in the literature. We compared the light curves of Venturi et al. (2001) and Teräsranta et al. (2005) with the outflow rate plots of our simulations to find a possible correspondence. The light curves of Teräsranta et al. (2005) are at higher frequency and span for 24 years, being more sensitive to the flux variability of the sources. Therefore, we adopted only light curves from Teräsranta et al. (2005). We performed a Fourier analysis of the simulated outflows and radio light curves data in order to compare the Fourier frequencies. The Fourier analysis allows a precise comparison of outflow frequencies with light curve frequencies of observed AGN, according to the hypothesis that outflow hydrodynamic frequencies are related to corresponding modulations in the AGN radio light curves. Moreover, the Fourier spectra allow us to resolve possible secondary periodicities. In Figs. 4 to 7, we report some examples of fit of light curve spectra (continuous lines) to outflow periodicities found in our models (dashed lines). For some sources, no good fit has been found between source periodicities and simulated ones. Instead, the superposition of simulated Fourier spectra to observed ones is quite good in some cases, suggesting that the hydrodynamic modelling should realistically describe the outflow power engine.

Outflow periodicities of simulated models can be converted into simulated Fourier frequencies to get a comparison with real light curve Fourier frequencies. Moreover, those sources whose observed periodicities fit the simulated outflow periods, offer a direct tool to link real periods in a light curve to simulated periods.

\section{Discussion and conclusions}

\subsection{Other models}

We will now compare this work with others in the literature. Our model can be considered as an axially symmetric, convectiondominated, inflow-outflow non-radiating solution. Such a modelling, because turbulent dynamics is fully resolved, is different from an advection-dominated, inflow-outflow solution (ADIOS, Blandford \& Begelman 1999, hereinafter BB99), where a nonradiating disc, showing radial motions, is described. Our approach also differs from that of BB99 in some other ways.

In our model, we assume only the azimuthal symmetry of all disc model properties, but we impose no restrictions to both outflowing mass rate and disc thickness. Moreover, we work out the simulation along the $z$-direction which, because of turbulence motions, needs small values of viscosity (usually $\alpha \leq 0.01$ ).

A more recent description of an ADIOS is shown in Blandford \& Begelman (2004, hereinafter BB04), where a twodimensional axisymmetric flow is considered, and the convection plays an important role. Although in our model convection is included, the horizontal advection is effective also on the disc surfaces, as is shown by plots where vertical advective outflows come out periodically near to the shocked disc zone. However, the BB04 model prevents any radial shock formation, both stationary or periodic. It is worth noting that BB04 discuss the possibility of a cycle similar to the one described in this work, although in their results the outflow is not an efficient mechanism in carrying out energy. Finally, Becker et al. (2001) extend the ADIOS model, including a pseudo-Newtonian potential, as is adopted in this work, and show that in their model an outflow comes out from a disc region closer to the SMBH with respect to that found by BB04, similar to what occurs in Lanzafame et al. (1998) for lower $\alpha$ values.

Also, other authors (Hawley \& Krolik 2001, 2002; McKinney \& Gammie 2002; McKinney 2006) have performed simulations of viscous accretion discs around black holes. These authors do not pay attention to a study of possible periodical outflows or the effect of the turbulent viscosity on periodicities. They consider the disc global structure in regimes mainly without shock fronts. Moreover, Hawley \& Krolik (2001, 2002) adopt very high $\alpha$ values, in the range $0.01 \div 10$, therefore excluding physical conditions able to develop either stationary or periodic shock fronts. Higher $\alpha$ values involve thinner accretion discs and less turbulent (more laminar) fluid dynamics, increasing both the radial component of flow velocity and the accretion rate at the same time. McKinney \& Gammie (2002) report that including the turbulent viscosity smoothes out any fluid discontinuity and supports a higher radial transport of fluid dynamical quantities. However, these authors do not use a rigorous Shakura and Sunyaev prescription. Instead, they assume that the viscosity is due to the development of magneto-rotational instability in the disc, calculating possible values of $\alpha$ in terms of such instability.

\subsection{This work}

The $\alpha$ values assumed in our models are fixed values in the range $\left(0 \div 4 \times 10^{-3}\right)$. These values are in agreement with the adopted viscosities in Lanzafame et al. (1998) in their study of steady shock formation in sub-Keplerian viscous accretion flows 

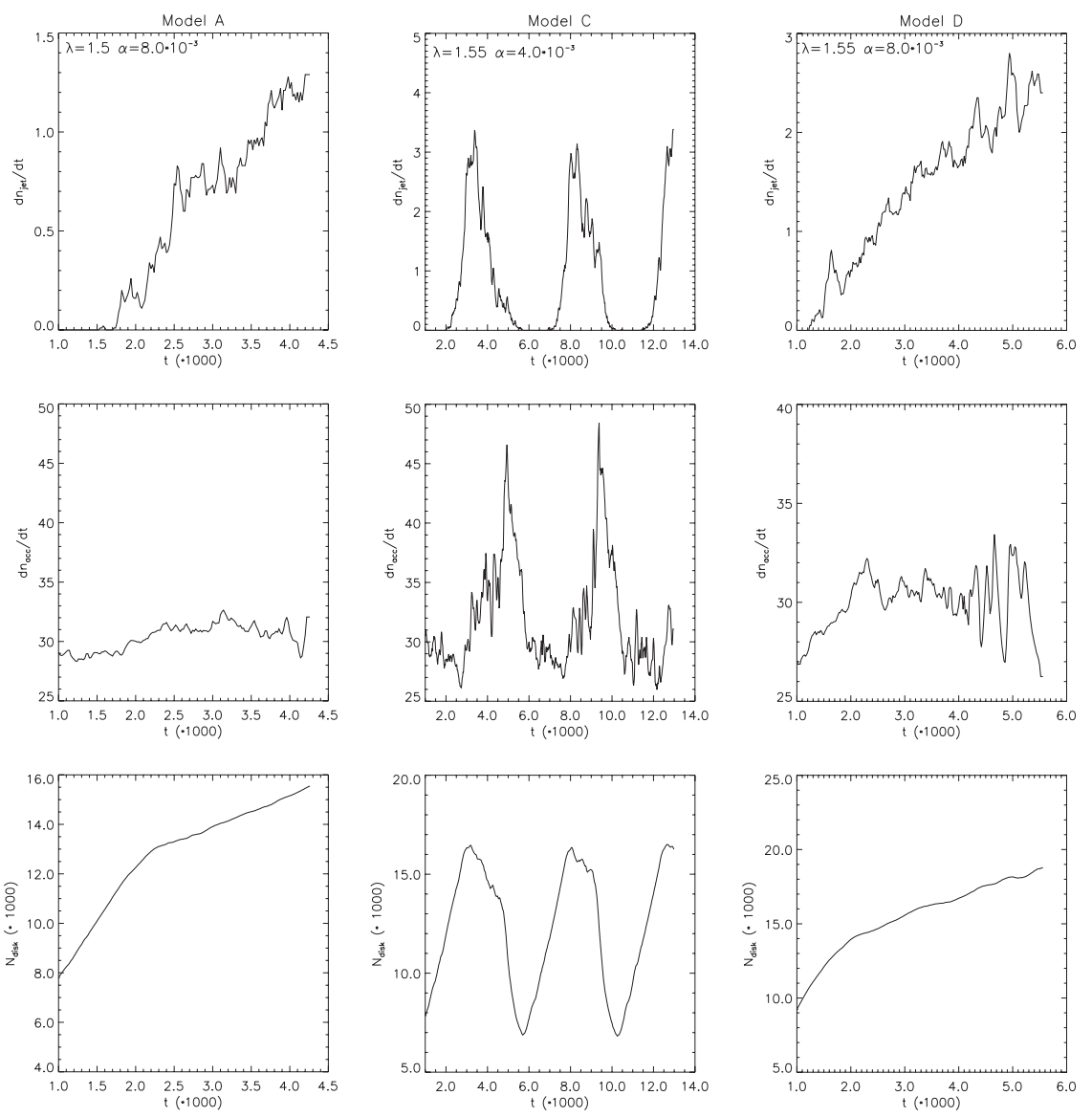

Fig. 2. Plots of the outflow rate $\mathrm{d} n_{\text {jet }} / \mathrm{d} t$, the accretion rate $\mathrm{d} n_{\text {acc }} / \mathrm{d} t$, and the number of particles in the disc $N_{\text {disk }}$ versus time. Quantities in the plots are expressed in simulation units. At the top of each panel, the adopted $(\lambda, \alpha)$ parameters and the model ID are also reported.
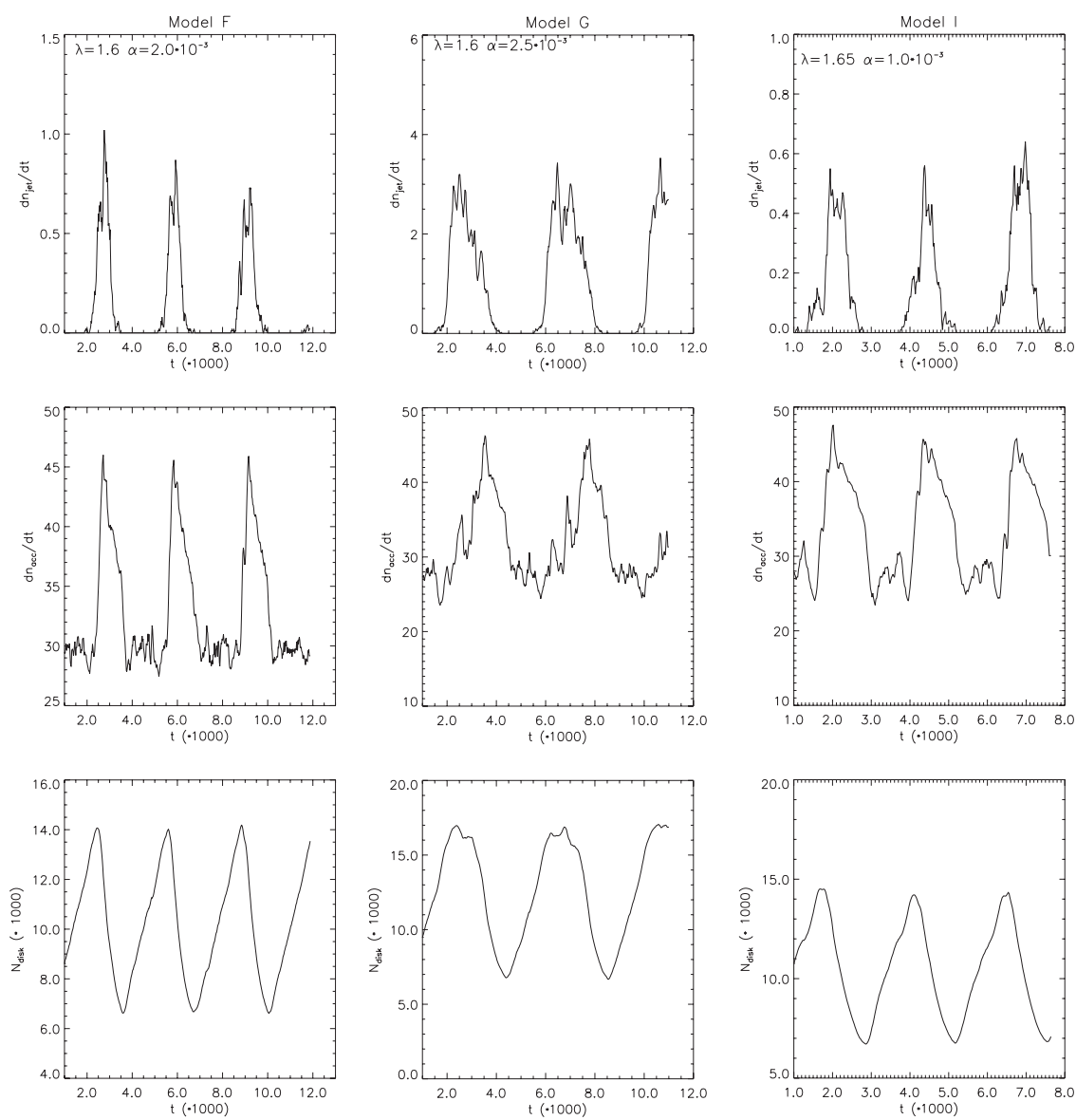

Fig. 2. continued. 

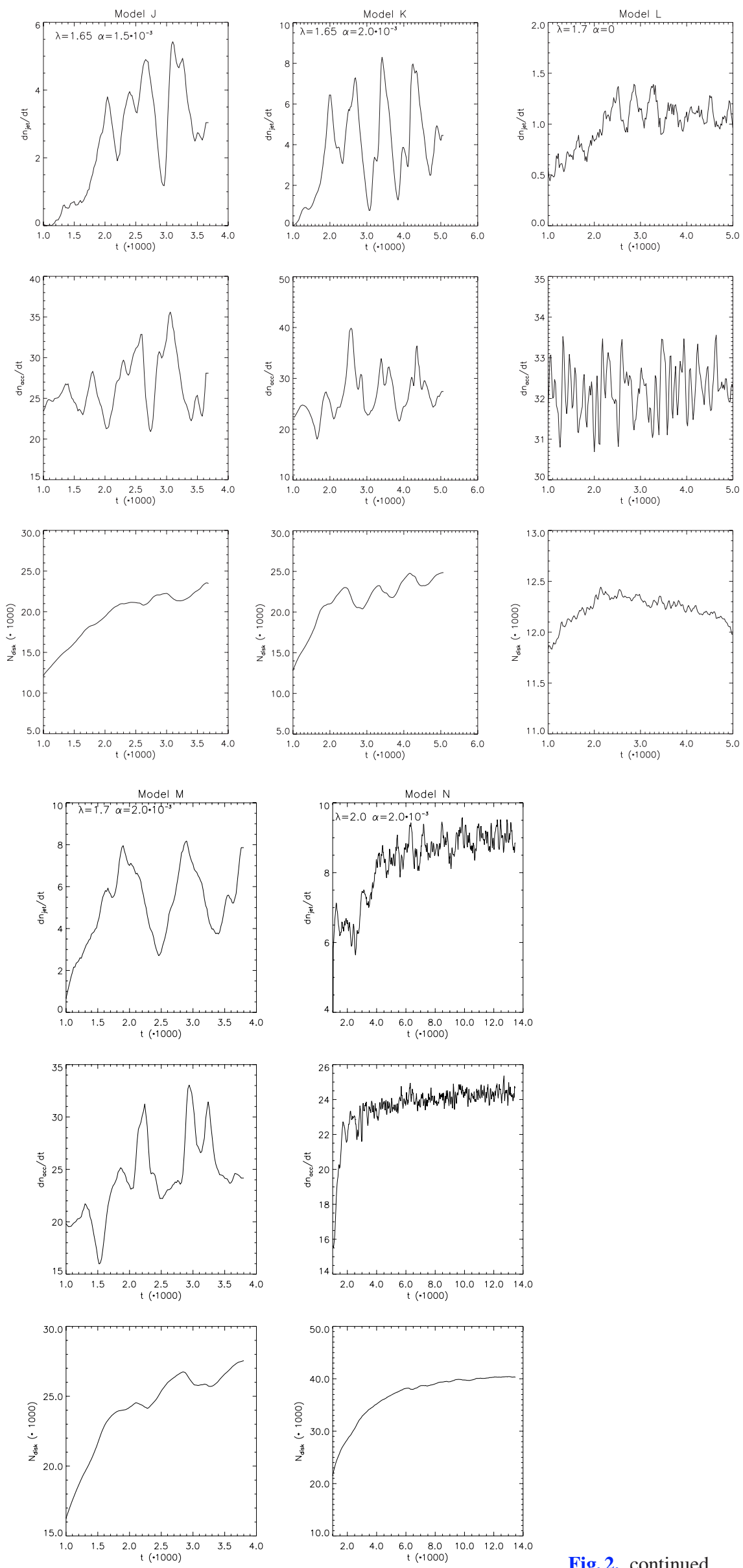

Fig. 2. continued.

Fig. 2. continued. 

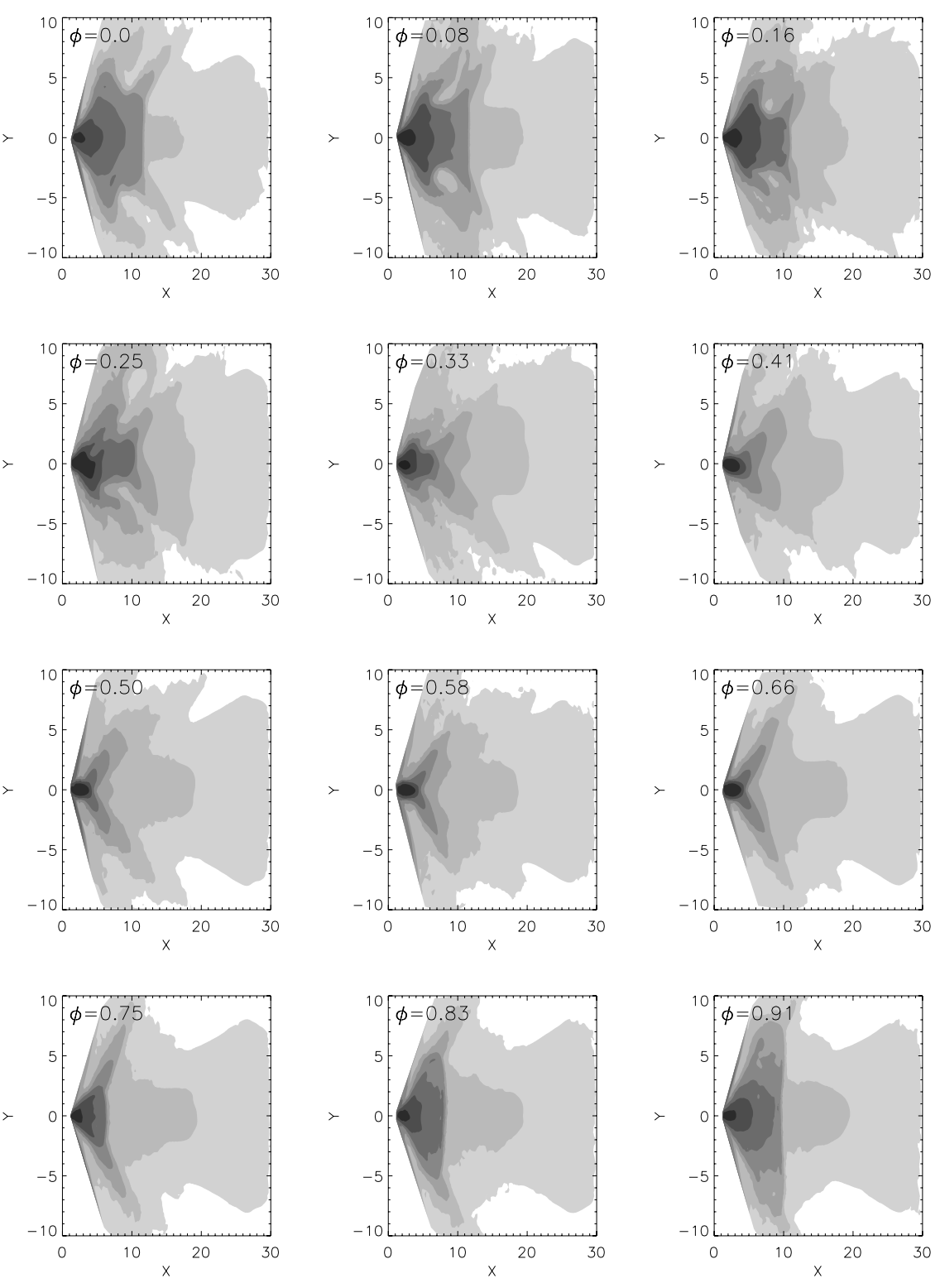

Fig. 3. Twelve $X Y$-plots concerning different phases $\phi$ of the periodical accretion disc time evolution with $\alpha=2 \times 10^{-3}$, and initial outer edge boundary conditions $v_{X}=0.1$ and $\lambda=1.6$. In such plots, density isocontours are shown. Distances are expressed in units of the Schwartzschild radius. For $\phi$ in the range $0 \div 016$, the $\mathrm{RH}$ conditions are satisfied. Instead, for $\phi$ in the range $\sim 0.16 \div 0.90$, the RH conditions are not satisfied and the shock front decays and then forms again. From $\phi \sim 0.90$ and afterward, the RH conditions are satisfied again and the shock front remains at $X \simeq 10$. onto a BH. These values are lower than those commonly assumed in other, previously-cited, AGN accretion viscous flow models. Higher viscosities degrade any shock front, disrupting any connection between outflows and radial shock periodicities. Instead, in our models, the two structures are strictly connected. According to our results, vertical outflows emerge from the subsonic post-shock regions whenever the mass transfer rate across the transonic region (the shock front) is higher than the mass accretion rate onto the $\mathrm{SMBH}$, due to the overcompression of the subsonic post-shock zone itself. In this context. a modest viscosity favours the radial flow without degrading the radial shock front too much. To test our model, we also performed simulations with $\alpha=0.1$ and $\lambda=1.3,1.6$. However, we do not discuss results of these simulations in this work because they are beyond the aims of this paper. In fact, in both cases, the shock front is smoothed out and the accretion continues without any outflow.

Our simulations have pointed out that for several values of the specific angular momentum $\lambda$ (at the outer edge as an initial boundary condition) and the $\alpha$ viscosity parameter, we observe the formation of an oscillating radial shock front. Moreover, this produces a periodical outflow of matter from the disc plane. Both periods agree with each other, and are mainly correlated to the radial disc dynamics in sub-Keplerian conditions as a consequence of the viscous radial transport. Therefore, such periodicities are not directly concerned with the disc inner edge rotation.

The whole hydrodynamic behaviour can be explained by considering that in the inner part of the disc, the angular momentum is transported outward as shown in Fig. 3, during phases $\phi$ within the range $0 \div 0.16$. In our simulations, the shock front develops at a larger distance from the SMBH, the larger is the adopted viscosity parameter, if the initial kinematic injection boundary conditions at the disc outer edge are fixed. When the shock forms, the RH conditions are not locally satisfied. This means that the shock front moves outwards, fixing its position at a radial distance where the density ratio between the preand post-shock regions satisfies the RH conditions. In the preshock region the matter continues to pile up, crossing the weak transonic shock and filling up the subsonic post-shock region. Whenever the mass transfer rate across the inner transonic shock is higher than the accretion rate onto the SMBH itself, or whenever it is higher than the mass transfer rate across another inner transonic shock, one or more outflows come out from the 


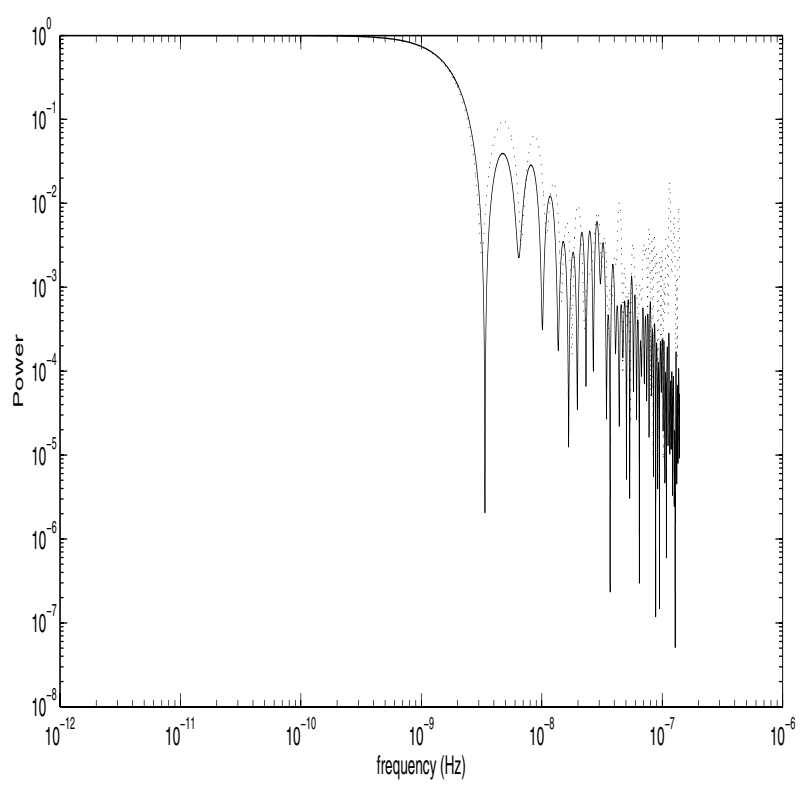

Fig. 4. Fourier spectrum of the flux time variation of 0440-003 (solid line) with superimposed Fourier spectrum of model L (dashed line). L is the model whose simulated Fourier spectrum better fits the source power spectrum. Frequencies are expressed in $\mathrm{Hz}$ and power is normalized to its maximum.

post-shock regions and the same transonic shocks disrupt and are dragged inward. When the density in the post-shock region increases too much, the RH conditions are no longer satisfied. During such phases the post-shock region of the disc is overcompressed and some matter is pushed out from the disc plane, this creating a "flare" in the outflow and vanishing the shock front. A quiescent phase follows, where the matter begins to pile up in the inner disc regions after some time, close to the centrifugal barrier peak position, producing a new shock front close to the $\mathrm{SMBH}$ in sub-Keplerian inflow conditions without any dissipation. In this way, the periodical radial motion of the shock front involves a periodical variation of the outflow rate. The simulations we carried out, and the previous results of Lanzafame et al. (1998), show the important role of the viscosity parameter in the origin and modulation of outflows in AGN. Indeed, although a periodical outflow can occur even in an inviscid flow, if $\lambda>1.7$, the viscosity modulates the periodicity. A particular combination of $\lambda$ and $\alpha$ gives a kind of "signature" to the periodicity of the light curve of each source, as is shown in Fig. 4. Therefore, it is possible to relate the physical conditions of the accretion disc to the periodical behaviour of the radio emission, having a powerful tool to study the accretion processes around a SMBH.

Working with a lower $\alpha$ parameter, in the range $0 \div 1.5 \times$ $10^{-3}$, Lanzafame et al. (1998) showed a correlation between the adopted $\alpha$ and the radial distance of the steady shock in subKeplerian initial conditions. In that paper, oscillations of the shock position were also found for $\alpha>1.5 \times 10^{-3}$, although not discussed. In this work, we pay attention to periodical oscillations and related outflows, and identify in the pairs $(\gamma, \alpha)$, in selected domains, the engine powering both periodical shock oscillations and related periodical single or multi-outflows.

Our model outflows can be correlated with the synchrotron radio emission of AGN because the ejected gas should be accelerated and collimated in a jet. In such a way, variations in the outflow from the disc surface, and consequently in the amount of gas channelled in the jet, are related to variations in the souce

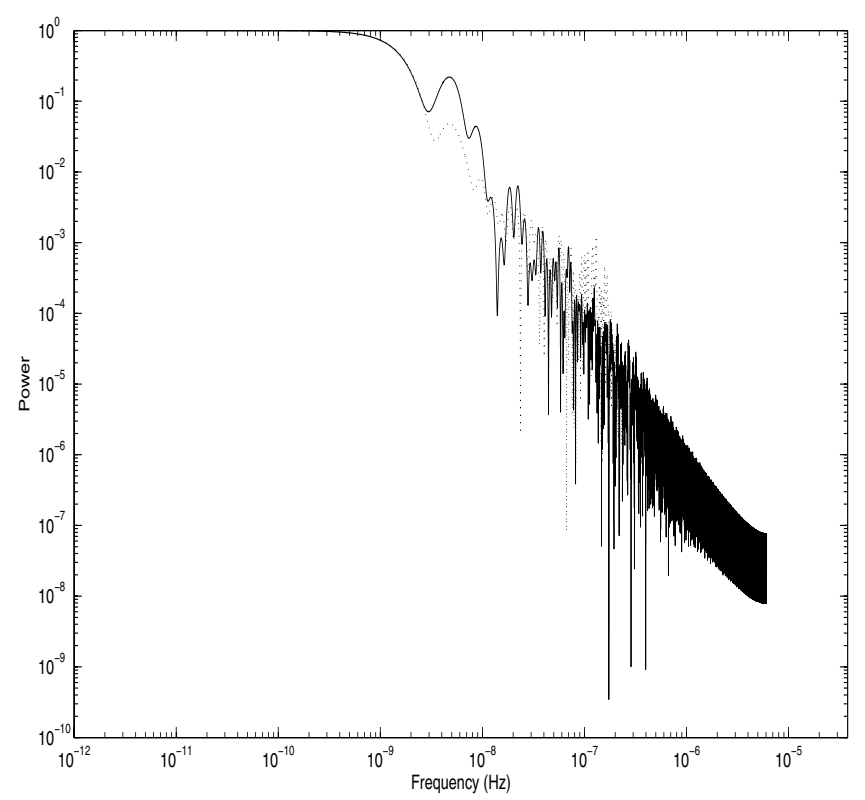

Fig. 5. Fourier spectrum of the flux time variation of 1730-130 (solid line) with superimposed Fourier spectrum of model A (dashed line). Scales are as in Fig. 4.

radio emission. We stress that a detailed description of the mechanism of jet formation and collimation is beyond the aims of this paper. Here we only note that a modulation of the outflow can affect the luminosity of the jet by simply varying the amount of matter involved. Such behaviour is consistent with the radio structure observed in high resolution images of radio loud AGN, where blobbed structure are common. Such a features could be related to a discontinuous ejection of matter. In low resolution images, the modulation of the gas ejection appears in a radio flux density variability, which is observed in many objects. In particular there are several studies of radio light curves of single objects (e.g., Vicente et al. 1996; Raiteri et al. 2001) or of group of blazars (e.g., Venturi et al. 2001). In latter works, it is possible to recognize a quasi-periodical variability of the sources, much more evident in the high-frequency light curves Teräsranta et al. (2005).

Figure 8 shows the rotation periods of the disc inner edge, the outer edge, and of the annular rings where radial shocks occur in comparison with the outflow periods (and the radial shock periods) for a selection of $(\gamma, \alpha)$ pairs of the simulated models. No statistically significant correlation is found among outflow periods and rotational periods. This shows that, even though the tangential dynamics has a role in the centrifugal barrier morphology, no strict correlation exists among the outflow periodicities (as well as the periods of the radial shocks) and the inner edge dynamics or the rotational dynamics of shocks.

Concluding, in this work we pay attention to the role of viscosity in order to parametrize a model of accretion disc onto a SMBH. Our models allows us to associate jets with mass ejection rates, without further information on the jet geometry. The results can be summarized as follows:

- the viscosity parameter determines the outflow regime;

- a correlation between viscosity and angular momentum with periodical behaviour of the blazars exists;

- the values of the viscosity parameter that produce periodic outflows are in a limited range. 


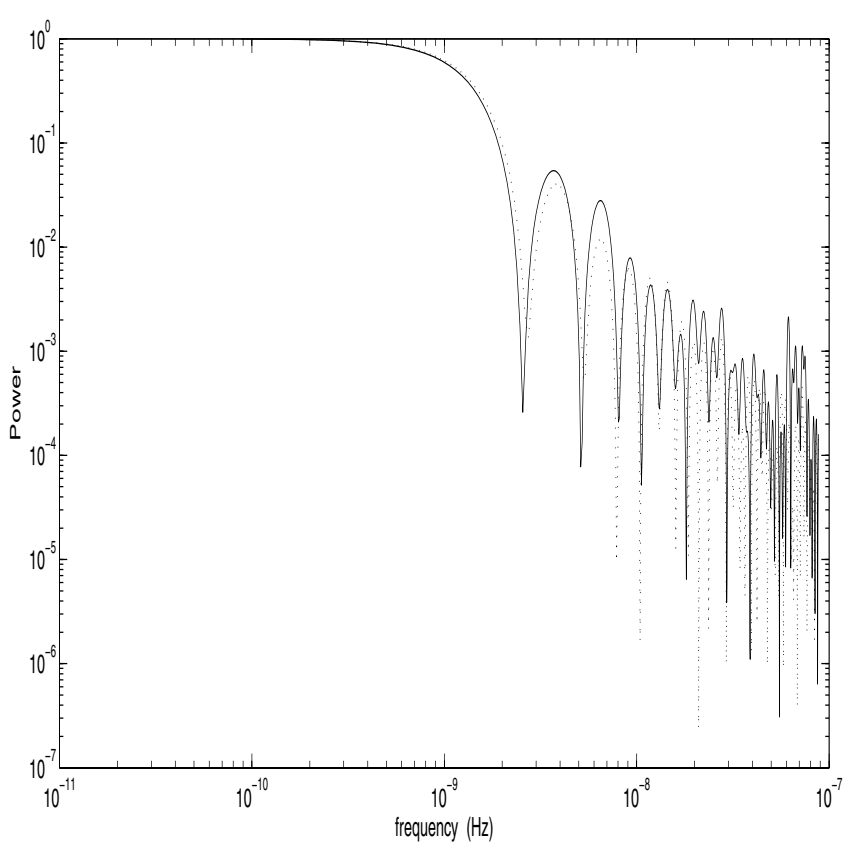

Fig. 6. Fourier spectrum of the flux time variation of 1101+384 (solid line) with superimposed Fourier spectrum of model $\mathrm{N}$ (dashed line). Scales are as in Fig. 4.

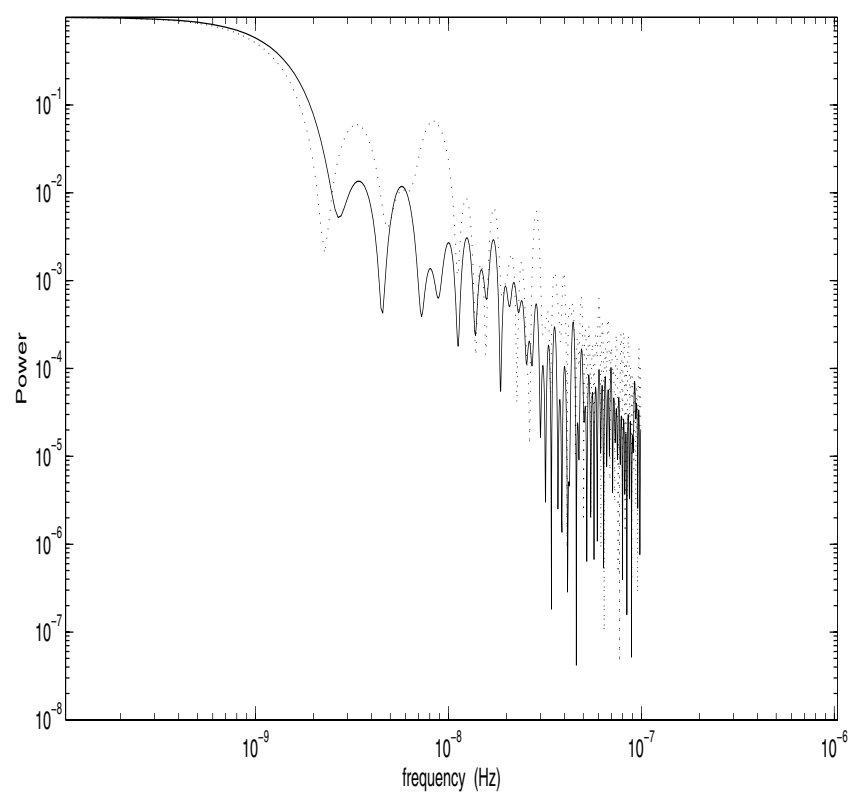

Fig. 7. Fourier spectrum of the time flux variation of $3 \mathrm{C} 279$ (solid line) with superimposed Fourier spectrum of model K (dashed line). In this case, the model whose simulated Fourier spectrum best fits the source is model K. Scales are as in Fig. 4.

We are well aware that in accretion discs, especially in AGN, the role of radiative processes and the interaction matter-radiation (heating, cooling, and transport) could be relevant. Of course, our models are purely hydrodynamic. Nevertheless, such models and their results are reliable in sub-Eddington conditions. According to Heinzeller \& Duschl (2007) the Eddington limit for an accretion disc is a linear function of the BH mass itself: $\dot{M}_{\mathrm{SMBH}, \mathrm{Edd}}=40 \pi G M_{\mathrm{SMBH}} / c$ in physical units. If $r$ is the particle accretion rate in our non-dimensional units (Fig. 2) - typically of the order of $20 \div 40$ particles/time - the physical accretion rate in our models is: $\dot{M}_{\mathrm{SMBH}}=19.12 \pi \rho r G^{2} M_{\mathrm{SMBH}}^{2} c^{-3}$. Therefore,

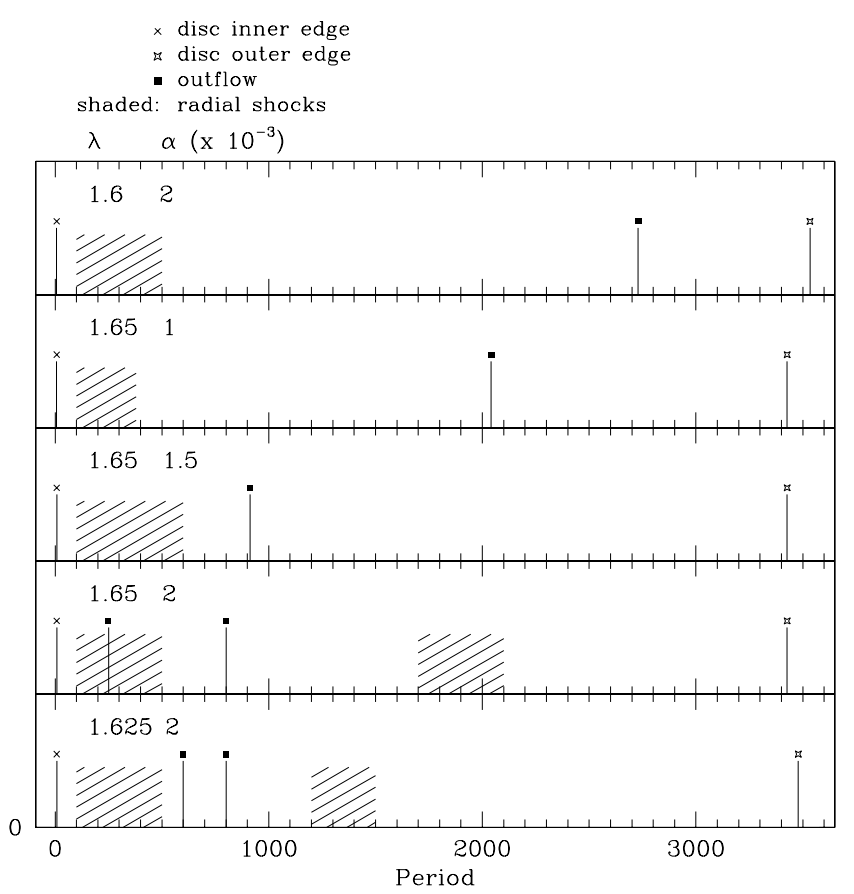

Fig. 8. The rotation periods of the disc inner edge, the outer edge and the annular rings where radial shocks occur in comparison with the outflow periods (as well as the radial shock periods) for five pairs of $(\gamma, \alpha)$. Shaded bars refer to the rotation periods of the annular rings where periodical radial shocks form and degrade in their cyclical evolution. Both outflows and radial shocks can be single or double.

since $\dot{M}_{\text {SMBH }}<\dot{M}_{\text {SMBH,Edd }}$, our pure hydrodynamic models are still valid. In such a case, we find an inverse correlation between the $\mathrm{BH}$ mass and the $r^{-1} \rho^{-1}$ term: $M_{\mathrm{SMBH}}<357 \times 10^{28} r^{-1} \rho^{-1}$ in cgs units.

As far as the role of the magnetic field is concerned, in recent years several papers have been written and very different hypotheses have also been suggested as far as the magnetic field magnitude, its morphology, and its boundary conditions are concerned. Results show that this topic is very far from any conclusion. However, in this paper, while we do not introduce any magnetic field effect, we can show that the simple hydrodynamic hypothesis is fully valid if, per unit mass, the magnetic energy is locally lower than the sum of the thermal and kinematic energies. Namely, if locally $B^{2} / \rho<c_{\mathrm{s}}^{2} /[\gamma(\gamma-1)]+v^{2} / 2$, strictly in the ideal case where the magnetic field is spatially ordered. This implies, according to our models that locally $B^{2} / \rho<$ $0.2 \div 0.4 \times 10^{21} \mathrm{erg} \mathrm{g}^{-1}$, hence $B<2 \div 3 . \times 10^{10} \rho^{1 / 2}$ in cgs units. In the case of a spatially disordered magnetic field, this lower limit is even higher because a disordered magnetic field is less effective in affecting the gas hydrodynamics.

Here we stress that our modelling is more concerned with the correlation between simulated periodic outflows and observed AGN jet periodicities than with jet formation and AGN emitted power. However, we suggest why this modelling may reproduce the observed power of AGN jets. Indeed, following Hughes \& Miller (1991), in the framework of our modelling the frequency distribution of the synchrotron spectrum $L(v) \simeq$ $m_{\mathrm{SPH}}^{\text {(blob) }} / M_{\odot} B_{\perp}^{(\delta+1) / 2} v^{-(\delta-1) / 2}$, where $m_{\mathrm{SPH}}^{(\text {blob })}$ is the typical SPH blob mass, $\delta \simeq 2.6$ and $B_{\perp}$ is the magnetic field component, normal to the line sight. For frequencies in the observed AGN radio spectra $v \sim 20 \mathrm{GHz}$, typically $L(v) \sim 10^{30} \div 10^{34} \mathrm{erg} \mathrm{s}^{-1} \mathrm{~Hz}^{-1}$, where the large spread in the monochromatic power mainly depends on the mass of the central accretor (affecting the mass accretion 
rate) and on the magnetic field magnitude, apart from other minor factors. If $v \sim 2 \times 10^{10} \mathrm{~Hz}, L(v) \simeq B_{\perp}^{(\delta+1) / 2}\left(m_{\mathrm{SPH}}^{\text {(blob) }} / M_{\odot}\right)$. Therefore, this evaluation clearly shows that the emitted power is due either to high magnetic field values or to high ejected blob mass values $m_{\mathrm{SPH}}^{\text {(blob) }}$ (according to our models), or to both. For instance, if $m_{\mathrm{SPH}}^{(\mathrm{blob})} \sim 10^{6} M_{\odot}$ and $B_{\perp} \sim 2 \times 10^{13}$ gauss, we get $L(v) \sim 10^{30} \mathrm{erg} \mathrm{s}^{-1} \mathrm{~Hz}^{-1}$. Notice that each ejected blob usually contains hundreds of SPH particles, so the average particle mass $m_{\mathrm{SPH}} \sim 10^{-2} \div 10^{-3} m_{\mathrm{SPH}}^{\text {(blob) }}$. Therefore, our models can explain the emitted AGN monochromatic power spectra with SPH particle masses of the order of $10^{3} \div 10^{4} M_{\odot}$.

We believe that these preliminary results justify further investigation. Therefore, new simulations will be carried out for different values of the viscosity parameter versus different values of the specific angular momentum of the injected particles, in order to better cover the $\alpha, \lambda$ parameter space, and therefore to have the possibility to compare with a larger number of light curves.

Acknowledgements. The authors thank an anonymous referee for carefully reading the paper and providing very useful comments and suggestions.

\section{References}

Blandford, R. D., \& Begelman, M. C. 1999, MNRAS, 303, L1 Blandford, R. D., \& Begelman, M. C. 2004, MNRAS, 349, 68 Blandford, R. D., \& Payne, D. G. 1982, MNRAS, 199, 883

Becker, P. A., Subramanian, P., \& Kazanas, D. 2001, ApJ, 535, 73

Bondi, H. 1952, MNRAS, 112, 195

Bondi, H., \& Hoyle, F. 1944, MNRAS, 104, 273

Bragg, A. E., Greenhill, L. J., Moran, J. M., \& Henkel, C. 1998, BAAS, 30, 1254

Camenzind, M., \& Krockenberg, M. 1992, A\&A, 255, 59

Chakrabarti, S. K. 1989, ApJ, 347, 365

Chakrabarti, S. K. 1990, Theory of Transonic Astrophysical Flow (Singapore: World Scientific)

Chakrabarti, S. K., \& Molteni, D. 1993, ApJ, 417, 671
Chakrabarti, S. K., \& Molteni, D. 1995, MNRAS, 272, 80

Chakrabarti, S. K., \& Das, S. 2004, MNRAS, 349, 649

Ferrarese, L., \& Ford, H. C. 1999, ApJ, 515, 583

Gomez, J., Marscher, A. P., Alberdi, A., \& Gabuzda, D. C. 1999, ApJ, 519, 642 Guerra, E. J. 2004, BAAS, 37, 381

Hawley, J. F., \& Krolik, J. H. 2001, ApJ, 548, 348

Hawley, J. F., \& Krolik, J. H. 2002, ApJ, 566, 144

Heinzeller, D., \& Duschl, W. J. 2007, MNRAS, 374, 114

Hughes, P. A., \& Miller, L. 1991, Introduction: synchrotron and inverseCompton radiation, Beams and Jets in Astrophysics, ed. P. A. Hughes, Cambridge Astrophysics Series, 1

Kellermann, K. I., Lister, M. L., \& Homan, D. C. 2004, ApJ, 609, 539

Königl, A., \& Choudhuri, A. R. 1985, ApJ, 289, 173

Lanzafame, G., \& Belvedere, G. 2005, ApJ, 632, 499

Lanzafame, G., Molteni, D., \& Chakrabarti, S. K. 1998, MNRAS, 299, 799

Li, Z., Chiueh, T., \& Begelman, M. C. 1992, ApJ, 394, 459

Lopez, J. A. 1997, Jets and Brets in Planetary Nebulae (Dordrecht, Kluwer), IAU Symp., 180, 197

Lucy, L. 1977, AJ, 82, 1013

McKinney, J. C. 2006, MNRAS, 368, 1561

McKinney, J. C., \& Gammie, C. F. 2002, MNRAS, 573, 728

Mirabel, I. F., \& Rodriguez, L. F. 1999, ARA\&A, 37, 409

Molteni, D., \& Sponholz, H. 1994, Mem. S. A. It., 65, 1183

Molteni, D., Lanzafame, G., \& Chakrabarti, S. K. 1994, ApJ, 425, 161

Monaghan, J. J. 1992, ARA\&A, 30, 543

Omang, M., Borve, S., \& Trulsen, J. 2006, JCoPh, 213, 391

Paczynski, B., \& Wiita, P. J. 1980, A\&A, 88, 23

Petschek, A. G., \& Libersky, L. D. 1993, JCoPh, 109, 76

Raiteri, C. M., Villata, M., Tosti, G., et al. 1999, A\&A, 352, 19

Raiteri, C. M., Villata, M., Aller, H. D., et al. 2001, A\&A, 377, 396

Reipurth, B., \& Bertout, C. 1997, Herbig-Haro Flows and the Birth of Stars, IAU Symp., 182 (Dordrecht, Kluwer)

Rieger, F. M. 2004, ApJ, 615, L5

Shakura, N. I. 1972, Astron. Zh., 49, 921 (English tr.: 1973, Sov. Astron., 16, 756)

Shakura, N. I., \& Sunyaev, R. A. 1973, A\&A, 24, 337

Tateyama, C. E., Kingham, K. A., Kaufmann, P., et al. 1998, ApJ, 500, 810

Teräsranta, H., Wiren, S., Koivisto, P., Saarinen, V., \& Hovatta, T. 2005, A\&A, 440, 409

Tout, C. A., \& Pringle, J. E. 1996, MNRAS, 281, 219

Venturi, T., Dallacasa, D., Orfei, A., et al. 2001, A\&A, 379, 755

Vicente, L., Charlot, P., \& Sol, H. 1996, A\&A, 312, 727 